OPEN ACCESS

Edited by:

Vladimir Litvak,

UCL Institute of Neurology, UK

Reviewed by:

Marcel Bastiaansen,

NHTV Breda University of Applied

Sciences, Netherlands

Ediz Sohoglu,

University College London (UCL), UK

${ }^{*}$ Correspondence:

Burkhard Maess

maess@cbs.mpg.de

Fahimeh Mamashli

fmamashli@mgh.harvard.edu

tThese authors have contributed equally to this work.

Received: 27 June 2016 Accepted: 04 November 2016 Published: 15 November 2016

Citation:

Maess B, Mamashli F, Obleser J, Helle $L$ and Friederici $A D$ (2016) Prediction Signatures in the Brain: Semantic Pre-Activation during Language Comprehension.

Front. Hum. Neurosci. 10:591. doi: 10.3389/fnhum.2016.00591

\section{Prediction Signatures in the Brain: Semantic Pre-Activation during Language Comprehension}

\author{
Burkhard Maess ${ }^{1 * t}$, Fahimeh Mamashli ${ }^{1,2,3 * \dagger}$, Jonas Obleser ${ }^{4,5}$, Liisa Helle 6,7 \\ and Angela D. Friederici ${ }^{2}$
}

\begin{abstract}
${ }^{1}$ MEG and Cortical Networks Group, Max Planck Institute for Human Cognitive and Brain Sciences, Leipzig, Germany, ${ }^{2}$ Department of Neuropsychology, Max Planck Institute for Human Cognitive and Brain Sciences, Leipzig, Germany, ${ }^{3}$ Department of Neurology, Massachusetts General Hospital, Athinoula A. Martinos Center for Biomedical Imaging, Harvard Medical School, Boston, MA, USA, ${ }^{4}$ Max Planck Research Group "Auditory Cognition", Max Planck Institute for Human Cognitive and Brain Sciences, Leipzig, Germany, ${ }^{5}$ Department of Psychology, University of Lübeck, Lübeck, Germany, ${ }^{6}$ Elekta Oy, Helsinki, Finland, ${ }^{7}$ Department of Neuroscience and Biomedical Engineering, School of Science, Aalto University, Espoo, Finland
\end{abstract}

There is broad agreement that context-based predictions facilitate lexical-semantic processing. A robust index of semantic prediction during language comprehension is an evoked response, known as the N400, whose amplitude is modulated as a function of semantic context. However, the underlying neural mechanisms that utilize relations of the prior context and the embedded word within it are largely unknown. We measured magnetoencephalography (MEG) data while participants were listening to simple German sentences in which the verbs were either highly predictive for the occurrence of a particular noun (i.e., provided context) or not. The identical set of nouns was presented in both conditions. Hence, differences for the evoked responses of the nouns can only be due to differences in the earlier context. We observed a reduction of the N400 response for highly predicted nouns. Interestingly, the opposite pattern was observed for the preceding verbs: highly predictive (that is more informative) verbs yielded stronger neural magnitude compared to less predictive verbs. A negative correlation between the $\mathrm{N} 400$ effect of the verb and that of the noun was found in a distributed brain network, indicating an integral relation between the predictive power of the verb and the processing of the subsequent noun. This network consisted of left hemispheric superior and middle temporal areas and a subcortical area; the parahippocampus. Enhanced activity for highly predictive relative to less predictive verbs, likely reflects establishing semantic features associated with the expected nouns, that is a pre-activation of the expected nouns.

Keywords: semantics, prediction, language, MEG, N400

\section{INTRODUCTION}

Language comprehension is a demanding process, which requires decoding of highly structured speech signals within very short time (Friederici, 2002; Pylkkänen and Marantz, 2003; Poeppel et al., 2008). Success of speech perception is increased through the combination of input-driven and prediction-based analysis (Strauß et al., 2013). Predictions are derived from both prior knowledge 
and contextual information (Rao and Ballard, 1999; Bar, 2007; Bendixen et al., 2009; Griffiths and Tenenbaum, 2011). These predictions are needed to achieve an optimal performance, both in general low level sensory processing as well as in higher cognitive processing such as speech perception. They do not stretch into some far future, they rather concern the present. According to predictive coding theory, our brain continuously anticipates current sensory input by transferring information from hierarchically higher to lower areas via top-down processing (Engel et al., 2001; Friston, 2003; Bar, 2009; Kiebel et al., 2009; Huang and Rao, 2011; Rauss et al., 2011). This reduces the processing demands at lower levels of hierarchy if the input matches expectations. If the prediction was wrong, performance will be less than optimal, as appropriate actions for the predicted input might have started already. However, in the case of weak or no predictions, no negative influence on the performance is to be expected.

Recently, modeling language processing using predictive coding theory has gained attention (David et al., 2011; Hickok, 2012; Sohoglu et al., 2012; Jakuszeit et al., 2013; Park et al., 2015; Lewis et al., 2016). Gagnepain et al. (2012) propose a predictive coding model of single spoken word recognition and show that superior temporal gyrus (STG) neurons represent the difference between predicted and heard speech sounds. Interestingly, instead of a direct competition between co-activated words they claim that alternative word completions compete via eliciting different predictions. Furthermore, researchers now also focus on the neural mechanism related to "predictive" processing itself (i.e., the context that generates the prediction). In a visual paradigm using magnetoencephalography (MEG) measurements Dikker and Pylkkänen (2013) found increased activation in left middle temporal and left inferior frontal gyrus (IFG) to pictures of objects (e.g., a picture of an apple) that were predictive for a specific word (e.g., apple) compared to pictures that were not predictive for the same word (e.g., a picture of shopping bag full of groceries). Fruchter et al. (2015) investigated the serial processing of adjective-nouns phrases using MEG and observed higher activity in the left middle temporal gyrus (MTG) in response to highly predictive adjectives in adjective-noun phrases. Using combined eye-tracking and functional magnetic resonance imaging (fMRI), Bonhage et al. (2015) revealed the neural substrate for syntactic word category prediction in a distributed network including cortical areas relevant for language processing as well as subcortical areas.

So far, predictions in language processing have been reported for many linguistic levels such as lexical, syntactic and sentential semantics (Kutas and Federmeier, 2000; DeLong et al., 2005; Bonte et al., 2006; Dambacher et al., 2006; Federmeier, 2007; Dikker and Pylkkanen, 2011; Obleser and Kotz, 2011; Van Petten and Luka, 2012; Brusini et al., 2015; Lewis and Bastiaansen, 2015). Previous findings suggest that all contextual information have an immediate impact on linguistic predictions (Hale, 2001; Demberg and Keller, 2008; Levy, 2008; Smith and Levy, 2013). Particularly, N400 responses were robustly found as being sensitive to variations in lexical-semantic prediction (Kutas and Hillyard, 1980, 1989; Federmeier, 2007; Lau et al., 2009, 2013a,b). It has been reliably observed that N400 amplitude is reduced following a predictive or supportive context (Kutas and Hillyard, 1984; Federmeier et al., 2007; Kutas and Federmeier, 2011; Wlotko and Federmeier, 2012).

The goal of the current study was the investigation of semantic context building during sentence processing. The main idea is that highly predictive verbs contribute more than average information towards the overall sentence meaning while the less predictive verbs provide less than average and keep a lot of options open. Consequently, the processing of the predicted nouns mainly constitutes of a confirmation whereas the processing of the less predicted nouns still involves at least the retrieval of semantic information and integration into the preceding context. While prediction formation has been investigated in the context of reading, thus far evidence from speech is less well-established. For this aim, we designed an MEG study based on a sentential N400 paradigm. In simple, spoken subject-verb-object sentences of German, we varied the predictability relation between the context (verb) and a sentence's final word (noun) as being high (e.g., He drives the car) or low (e.g., He gets the car). We used identical final determiner-noun phrases together in both contexts. Following highly predictive verbs, the presented noun is the one with highest cloze probability (Taylor, 1953). Following less predictive verbs, the (same) noun is one of many alternatives, however, all alternatives had equally low (or even lower) cloze probabilities. Consequently, none of our nouns is violating a prediction from the preceding context.

So far, using MEG, several approaches were taken in identifying the brain network underlying the N400m. In summary, the results suggest a left hemispheric dominance and the involvement of temporal and inferior frontal sources (Halgren et al., 2002; Marinkovic et al., 2003; Pulvermüller et al., 2005; Maess et al., 2006; Pylkkänen and McElree, 2007; Salmelin, 2007; Dikker and Pylkkänen, 2013). In addition, previous N400 studies also reported activity of deeper structures during the processing of semantics; these were perirhinal and inferior temporal structures active in semantic aspects of word recognition (Halgren et al., 2006) as well as thalamic nuclei responding specifically to semantic violations (Wahl et al., 2008).

We hypothesize that the previously shown reduction of the N400 for the highly expected words is mostly a consequence of the prior prediction formation during the processing of earlier presented semantic context. Less expected words need more processing effort and therefore produce a larger evoked response, because, more semantic information needs to be retrieved. Violations of the preceding context are not discussed here as such words may cause a more or less extensive error handling which are special cases in speech processing.

\section{MATERIALS AND METHODS}

\section{Stimuli}

We selected short German sentences in which the verb was either highly predictive (e.g., he drives the car, German: Er fährt das Auto) or less predictive (e.g., he gets the car, German: Er kriegt 


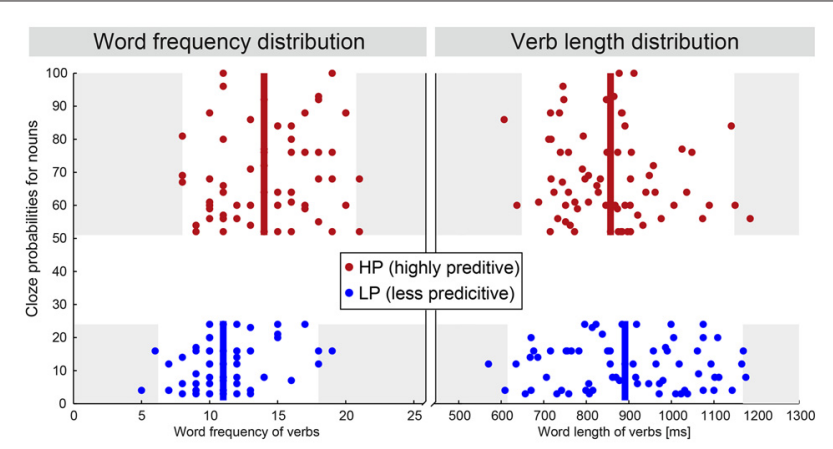

FIGURE 1 | Distribution of the word frequency and word length values for the verbs in both conditions. Both differences are small and therefore considered irrelevant for the effects discussed here. For more details see "Materials and Methods" Section-Stimuli. The white area marks the centered 95\% percentile.

das Auto) of the following noun. Stimuli were taken partially from an earlier study (Gunter et al., 2000) and an additional behavioral pre-study. In both cases participants had to fill in a printed form which provided a personal pronoun (Er/Sie [he/she]) followed by a verb. Participants were asked to complete the sentence beginnings in a simple way by providing just a determiner and a noun. During the analysis, we identified pairs of verbs falling in either of the two classes-highly predictive (cloze probability $>50 \%$ ) or less predictive (cloze probability $<25 \%$ ) of the following noun as previously done by Gunter et al. (2000). For the purposes of the present study, all stimuli were additionally controlled for their word frequencies ${ }^{1}$ and word length (Figure 1). The complete set of stimuli consists of 69 pairs. Sentences could be interpreted literally-a figurative interpretation is unlikely. As we use the same noun phrase for each of the sentence pairs-there are no stimulus driven differences at the noun level between both conditions. The verbs, however and intentionally, consist of two different groups. The median word frequency between both verb groups is different by a value of 3 which corresponds to a ratio 1:8. Halgren et al. (2002) observed a minor influence of word frequency towards the N400 when comparing mean word frequencies of 15 with 336 per million, which corresponds to a ratio of about 1:23. We have analyzed a subset of 39 pairs of our stimuli, which had no difference in the median word frequency nor in word length. We did not observe a change in the evoked condition differences between both stimulus sets. The set with 69 pairs had a higher signal-to-noise ratio though; hence we based the following analysis on the complete set of stimuli. Sentences were spoken by a trained female speaker at natural speed. Loudness was adjusted so that all sentences were at the same perceived level.

\section{Participants}

In total, 21 German native speakers (11 female) took part in an MEG experiment (age range: 20-32 years, median: 27). All were right-handed according to the Edinburgh Handedness

\footnotetext{
${ }^{1}$ http://wortschatz.uni-leipzig.de/
}

Inventory (Oldfield, 1971). None of them had a hearing deficit or neurological diseases. Participants gave written informed consent prior to the experiment and were paid for their participation. The study follows the guidelines of the declaration of Helsinki and has an ethical approval of the ethics commission of the University of Leipzig.

\section{Design and Procedure}

Participants were seated in a dimly lit shielding room (Vacuumschmelze Hanau, Germany). MEG data was recorded with a 306 channel Vectorview device (Elekta Oy, Helsinki, Finland) at $500 \mathrm{~Hz}$ sampling rate utilizing a bandwidth of $160 \mathrm{~Hz}$. Additionally, three bipolar channels were recorded to monitor eye and cardiac activity. Electrooculogram (EOG) electrodes were attached to the outer canthi and above and below the left eye. Electrocardiogram (ECG) electrodes were placed at the right clavicle and the left ribs. The experiment was conducted in one session with five recording blocks. First, participants' individual hearing thresholds were determined for both ears separately. Stimuli were presented at $48 \mathrm{~dB}$ SL (sensation level, i.e., above the individually determined hearing threshold). Sensation levels were estimated using a subpart of one sentence. Stimuli were randomized and presented once over two blocks, thereafter presentation of all stimuli was repeated in two further blocks using a different randomization. The onsets of all sentences, as well as the onsets of the verbs and the nouns in each sentence were specifically marked (Figure 2). During the fifth block participants heard a sequence of sinusoidal tones. Data of this block are not presented here.

Participants were instructed to listen carefully to the presented sentences and not to move during a block. They were asked to fixate their view at a cross at the center of the screen while listening to the auditory stimulation. Fixation crosses were presented from $700 \mathrm{~ms}$ before onset until $700 \mathrm{~ms}$ after the offset of each sentence. Fifteen percent of the sentences were followed by either the same or an alternative sentence spoken by a different voice (male). Participants' (incidental) task was to judge whether the two succeeding sentences (female and male voice) were the same. Participants responded by a button press following the cue. The cue also informed participants of the response-to-buttonalignment: it comprised of one happy and one sad symbolic face

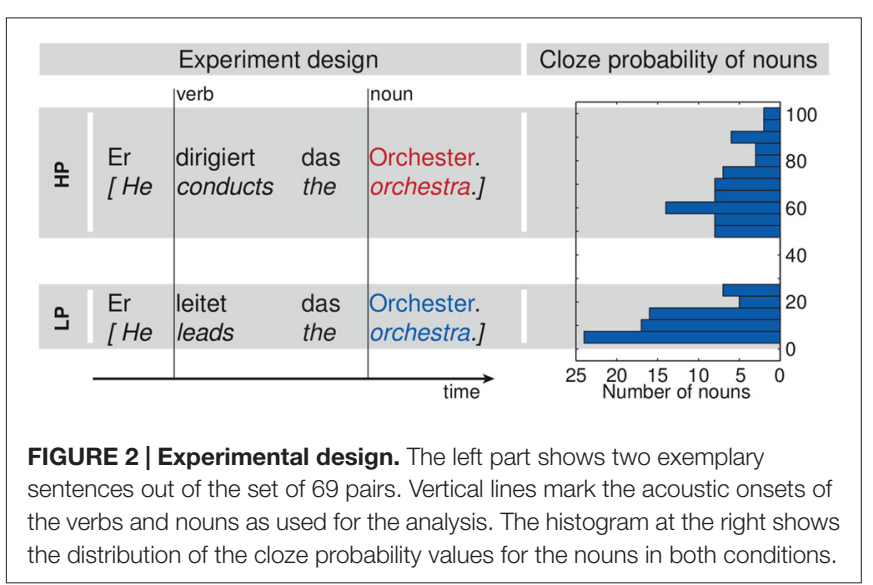


(smiley), one presented on either side of the screen. Participants had to use their thumbs to press either the left or the right side button, i.e., giving the answer "yes" with the button at the side of the happy face and "no" with the other. The arrangement of the faces on the screen was randomized and counterbalanced over all stimuli in each block.

\section{Data Preprocessing}

MEG data were corrected for head movements and external interferences by the Signal Space Separation (SSS) method (Taulu et al., 2005) implemented in MaxFilter ${ }^{\mathrm{TM}}$ software (Elekta Oy). Additionally, a recently developed SSS-based method (Taulu et al., 2012) for suppression of the uncorrelated sensor noise and artifacts was applied prior to SSS-processing to further enhance the signal-to-noise ratio. The MEG signals were filtered $(0.2-9 \mathrm{~Hz})$ to focus on the evoked, broad N400 deflection, as similarly done in e.g., (Maess et al., 2006; David et al., 2011). The high-pass filter (4367 points FIR, hamming window) was specifically designed for a strong DC suppression (>125 dB at DC) to replace the baseline correction. High-pass filtering was used instead of the classical baseline correction, because there is no "clean" time interval in the middle of a sentence when using normal connected speech as stimulus. Data was subjected to a spatial independent component analysis (ICA) decomposition using MNE python (Gramfort et al., 2013). ICA components, which showed a high temporal correlation with EOG or ECG channels and with a typical topography, were manually identified and removed before reconstructing the MEG signals. Two to five ICA components were excluded with a median of 3 over all subjects and blocks. Subsequently, epochs were selected as $1500 \mathrm{~ms}$ intervals (from $-300 \mathrm{~ms}$ to $1200 \mathrm{~ms}$ with respect to the onset of verbs or nouns, respectively). Epochs during which the standard deviation of signal channels within a sliding $200 \mathrm{~ms}$ time window exceeded either $200 \mathrm{pT} / \mathrm{m}, 5 \mathrm{pT}$ or $150 \mu \mathrm{V}$ (for gradiometers, magnetometers and EOG, respectively) were excluded from further analysis. Epochs were averaged into four categories: highly predictive verbs, less predictive verbs, highly predicted nouns and less predicted nouns. For simplicity, we use the same acronyms when labeling verb and noun conditions: HP means either highly predictive or highly predicted when used as either verb or noun condition label. LP (less predictive/predicted) is the counterpart. Preprocessing was performed using the MNE toolbox (Gramfort et al., 2014) and own Matlab ${ }^{2}$ routines. Evoked fields were subjected to source localization using the MNE toolbox (Gramfort et al., 2014) and finally mean sLORETA estimates of a total of nine selected cortical regions were estimated. Cluster analyses (Maris and Oostenveld, 2007) were conducted to justify the selections of the channels and the interval in time (see Figure 3). Evoked responses for the verbs and the nouns over all magnetometers and over the complete time interval from $-300 \mathrm{~ms}$ to $1200 \mathrm{~ms}$ were subjected to two cluster analyses comparing HP vs. LP. Since condition (HP/LP) is a within-subjects-variable-a two-sided paired $t$-test ("depsamplesT") was used for the generation of clusters $(\alpha$-threshold $=5 \%)$. Clusters "clusterstatistic $=$ maxsum;

\footnotetext{
${ }^{2}$ http://www.mathworks.com/
}

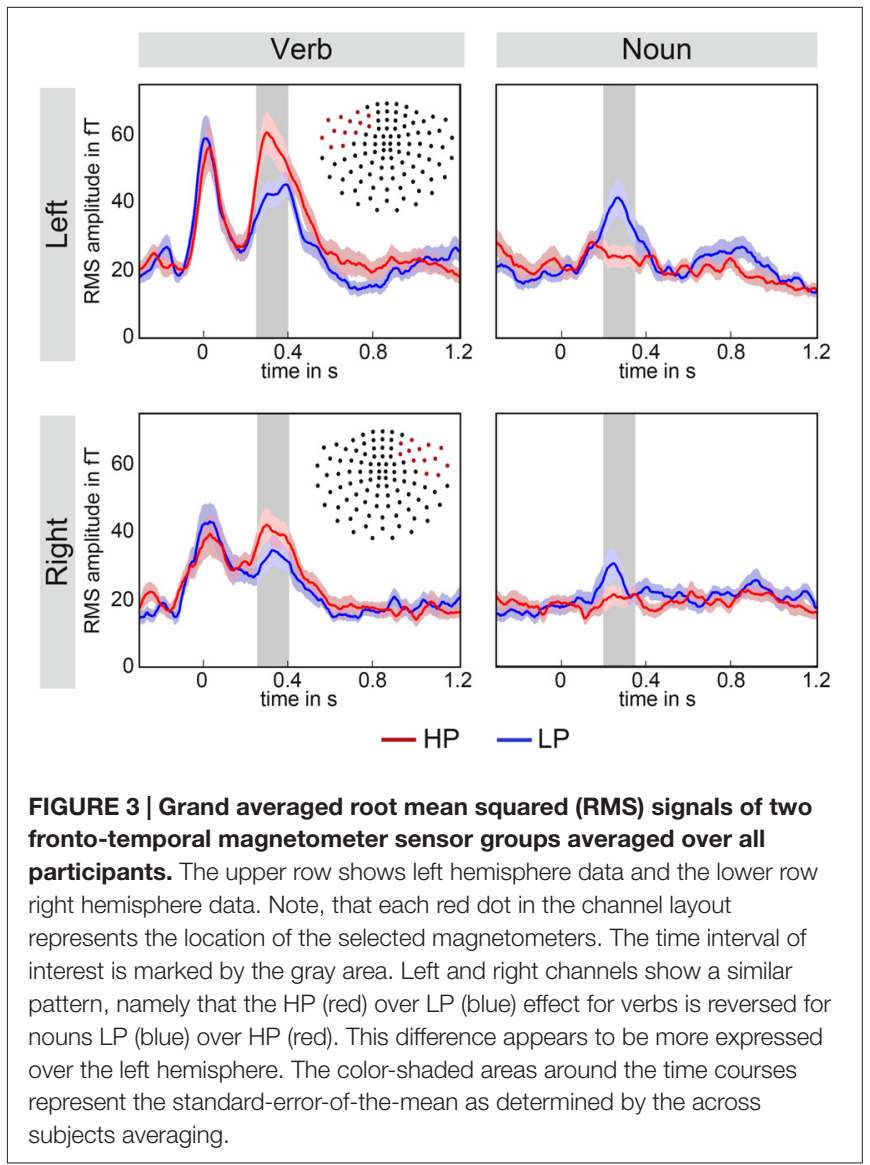

minnbchan $=2$ " were statistically tested using Monte-Carlorandomizations $(N=5000$, "correctm $=$ cluster") and a statistical threshold of $1 \%$.

\section{Source Localization}

Source localization of the evoked responses was performed using sLORETA minimum-norm estimation (Hämäläinen and Ilmoniemi, 1994; Pascual-Marqui, 2002), implemented in MNE Software ${ }^{3}$. sLORETA for cortically constrained surfaces results in noise-normalized activity values ( $z$-values). The sign of this sLORETA solution equals the sign of the current density estimated as minimum norm estimate at the same vertex. sLORETA regularization was set to an SNR value of 3 . Co-registration was based on the digitized head shape and the fiducial points. Individual anatomical $\mathrm{T} 1$ weighted $\mathrm{MR}$ images were segmented using Freesurfer 5.1.0 ${ }^{4}$. Based on this segmentation, single compartment boundary element models (BEM) were constructed and source spaces were provided as a set of current dipoles located within and mostly perpendicular to the cortical surfaces (loose value $=0.2$, which means that the source covariance matrix entries which correspond to the current surface normal are set equal to one and the two others are set equal to 0.2). Furthermore, individual cortical surfaces were

\footnotetext{
${ }^{3}$ www.nmr.mgh.harvard.edu/martinos/userInfo/data/MNE_register

${ }^{4}$ surfer.nmr.mgh.harvard.edu
} 
morphed onto the cortical surface of one participant to allow group averaging of the sLORETA estimates (Dale et al., 1999). ROIs were selected as subparts of regions defined by a Freesurfer parcellation (Destrieux et al., 2010). We selected nine regions per hemisphere known as parts of the network for semantic language processing (Lau et al., 2008; Price, 2010; Friederici, 2011), namely the primary auditory cortex (PAC), the planum polare $(\mathrm{PP})$, the planum temporale (PT), the anterior and posterior parts of the MTG (aMTG and pMTG), the temporal pole (TP), the IFG including Brodmann areas BA44 and BA45 and the parahippocampal gyrus aPH and $\mathrm{pPH}$. ROI magnitudes were computed as the mean of the absolute sLORETA values within each of the pre-selected ROIs. Two-way ANOVAs were conducted for each of the nine ROIs with the factors HEM (hemisphere) and PRED (predictability).

\section{RESULTS}

\section{Sensor-Level Analysis of The N400 Effects}

For sensor-level group analysis, the root mean squared (RMS) signal of a group of channels over left and right frontotemporal regions was computed (see Figure 3). RMS signals were estimated for gradiometers and magnetometers and for the verbs and the nouns, separately. Time range and channel selection were made in accordance with current literature which identified early, fronto-temporal N400 components when using MEG, see for instance (Pylkkänen et al., 2002; Maess et al., 2006). Analysis of the responses to the nouns (see Figure 3, right column) showed, as expected, a significant N400m effect observed for both sensor types-we present the values for the magnetometers only (LP-HP Left: $p<0.001, t_{(20)}=-4.25$; Right: $\left.p<0.05, t_{(20)}=-2.07\right)$ in the time window $200-350 \mathrm{~ms}$ with a larger amplitude for less predicted nouns. Most interestingly, at the preceding verbs (see Figure 3, left column) a significantly larger amplitude was observed for highly predictive compared to less predictive verbs (Left: $p<0.001, t_{(20)}=4.26$; Right: $p=0.005$, $\left.t_{(20)}=3.15\right)$ in the time window $250-400 \mathrm{~ms}$. Note that the time intervals, represented as gray areas in the figures, for the verb and noun tests were equal in length, but shifted by $50 \mathrm{~ms}$ towards earlier latencies for the nouns.

Cluster analyses were conducted for an independent justification of the selection of channels and time interval (Maris and Oostenveld, 2007). For the verbs, significant positive magnetic field differences were observed for two intervals in time-the first roughly located between $1000 \mathrm{~ms}$ and $1200 \mathrm{~ms}$ and the second between $250 \mathrm{~ms}$ and $400 \mathrm{~ms}$. The average length of the verbs is about $900 \mathrm{~ms}$ (see Figure 1), therefore only the earlier interval is relevant for the processing of verbs. This cluster mainly included left fronto-temporal
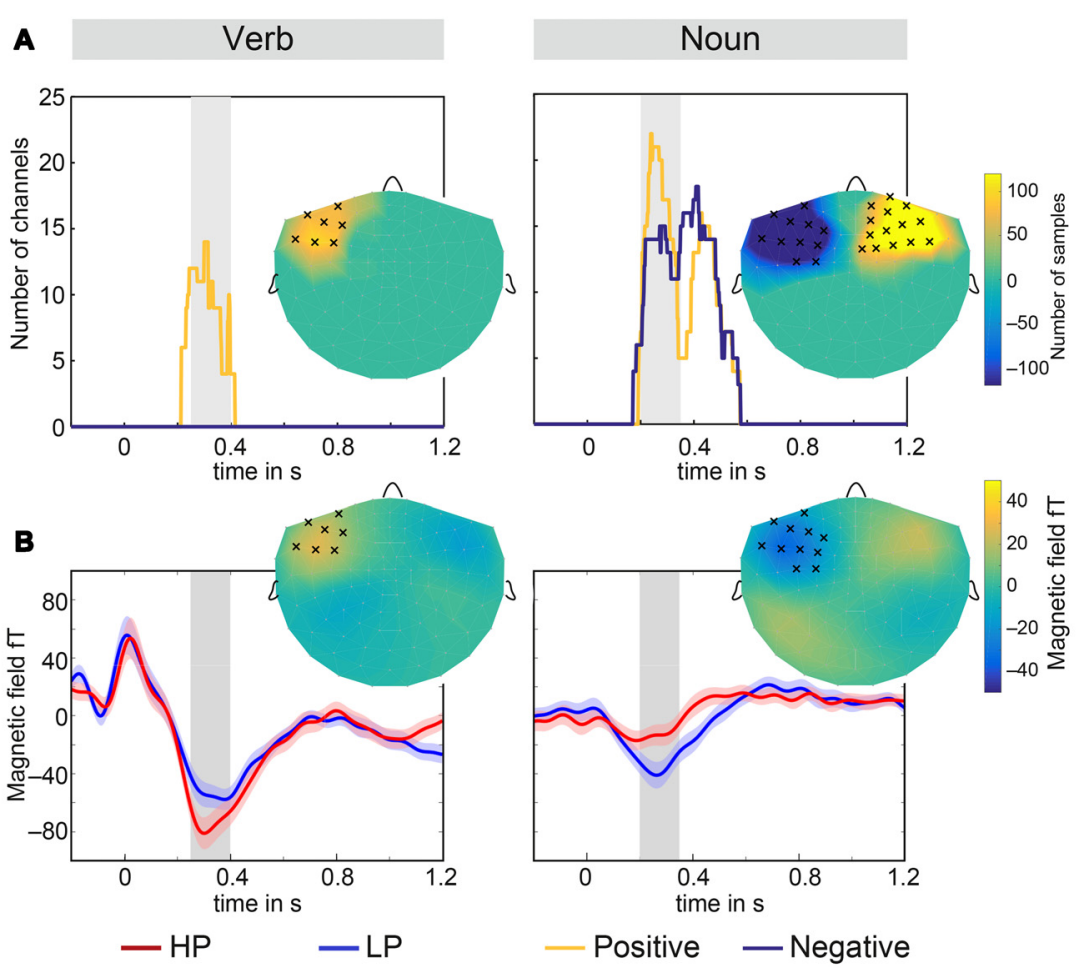

FIGURE 4 | Results of the cluster analysis. (A) Spatio-temporal extent of the three clusters for verbs (left) and nouns (right). The gray bar in the background marks the temporal intervals which were used throughout this article. The inserts show topographically the full number of samples in a cluster. Channels which had 50 or more cluster samples within the gray intervals were marked by crosses. (B) Average time courses of left hemispheric magnetometers in clusters for verbs and nouns and both conditions LP and HP. The shaded area around the solid lines displays the standard error of the mean. The inserts show topographically the magnetic field difference LP-HP averaged over the time interval marked in gray. Marked channels have 50 or more cluster samples within the gray interval. 
channels (see Figure 4A left). No significant effect for negative differences (right hemisphere) were observed. For the nouns, a positive and a negative cluster were observed both stretching in time from about $200 \mathrm{~ms}-500 \mathrm{~ms}$ (see Figure 4A right). Clusters included both left and right fronto-temporal channels. Therefore, the selection of channels and time interval in the RMS signal $t$-tests was highly overlapping with the cluster analyses results. Average time courses of significant channels within a cluster in the left hemisphere as well as the magnetic field difference (LP-HP) topography for verbs and nouns is shown in Figure 4B.

Sensor analysis in general is prone to a head-position bias: if heads were systematically closer to the left side of the dewar then sensors at this side also showed stronger responses. We therefore refrain from formulating arguments concerning the left-right differences of the above analysis and rather refer to source space analysis instead. Source analysis included the complete set of MEG sensors (gradiometers and magnetometers) and is therefore independent of the RMS channel selection or the channels in clusters, respectively.

\section{Source Localization of The N400 Effects}

To compare the spatial distributions of the sLORETA estimates for both the verb and the noun time intervals, the signed sLORETA estimate was additionally computed: here the projection to the surface normals of the folded cortex replaces the magnitudes. Individual estimates were then morphed onto the cortex of one of our participants. Figure 5 displays the grandaverage (mean across subjects) for the two conditions (HP and LP) at two latencies ( $350 \mathrm{~ms}$ for verbs, $300 \mathrm{~ms}$ for nouns). Three of the four panels show larger, left temporal estimates. Note, that the polarity and the location of the estimates are very comparable between all three panels. These single condition

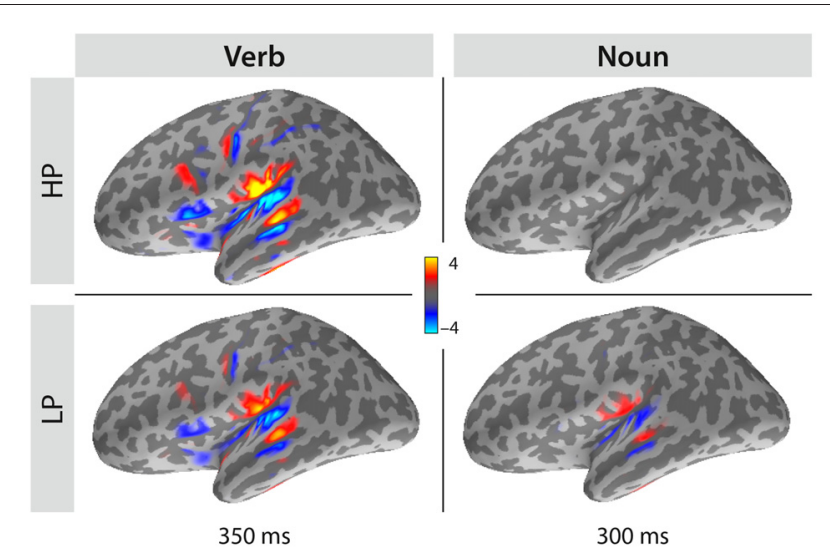

FIGURE 5 | Grand average sLORETA minimum norm source localization results projected to surface normals for both conditions (HP and LP) and both word classes (verb and noun) computed for latencies of $350 \mathrm{~ms}$ or $\mathbf{3 0 0} \mathbf{~ m s}$, respectively. Note, that the distribution of estimates is spatially very equivalent although it differs in strength. Interestingly, there is a reversal between conditions: highly predictive verbs (HP) elicit stronger responses than less predictive verbs (LP), but less predicted nouns (LP) elicit a stronger response than highly predicted nouns (HP). panels demonstrate that conditions mainly differ in strength, but not in spatial distribution. Computing the difference LP-HP (not displayed in the figure), which is the classical N400 in case of the nouns, leads to a similar picture as shown in the lower right panel. Computing LP-HP for the verbs, leads to a polarity reversal as HP has the stronger estimates compared to LP but otherwise very similar spatial distribution. We interpret this observation such that sentence processing asks for a certain amount of computational demands, which can be delivered at either the verb level, when the verb is highly predictive, or at the noun level, at the latest. Since the activity at the verb level is overall stronger than activity at the noun level, we would argue that recognizing a verb and setting up a meaningful context for it, needs some extra processing demands not needed at the following noun level. This is plausible as verbs may additionally generate specific syntactic restrictions, i.e., objects with special case markings. Less predictive verbs must provide less information than highly predictive verbs as the first do not allow specifying the next word. Lesser information, lesser processing demands, hence we expect a smaller evoked response for them. The same holds for highly predicted nouns which do not add much new information, because they are predicted-therefore their processing is finished early by about $250 \mathrm{~ms}$.

Mean sLORETA estimates were computed for each of the nine regions in both hemispheres as the mean over all estimates within a region. Figure 6 left panels (bar graphs) display the sLORETA estimates for those regions which showed a significant main effect of the condition (HP vs. LP) for both word classes (verb, noun). The bar graphs also show the standard error of the mean as a short black line at the top of each bar. Note that the sign of the difference always reverses when comparing the verb and the noun condition difference. Figure 6 right panels visualize the location and extent of each region with a significant effect overlaid of lateral or medial views of the inflated left hemisphere.

Figure 7 visualizes the across-participants correlation between the verb and the noun effect, that is difference LP-HP for both intervals. The dots in the scatter plots in the two left columns present single participant data. The line is estimated via linear regression of the verb and the noun effect. We have computed the regression for each of the nine cortical regions separately and in Figure 7, we show the data of those six regions in which a significant correlation was observable. Raw $p$-values are given within each of the panels. All regions, but pMTG and PAC are significant when correcting for multiple comparisons by factor of 18 (Bonferroni). pMTG and PAC have $p$-values of 0.14 and 0.11 after correction, respectively.

\section{DISCUSSION}

The neural signature of prediction during language comprehension was investigated using MEG sensor level analysis and sLORETA estimates. These revealed the expected large N400 amplitude for the less predicted (unexpected) nouns compared to predicted (expected) nouns; very consistent with previous studies using similar sentences 


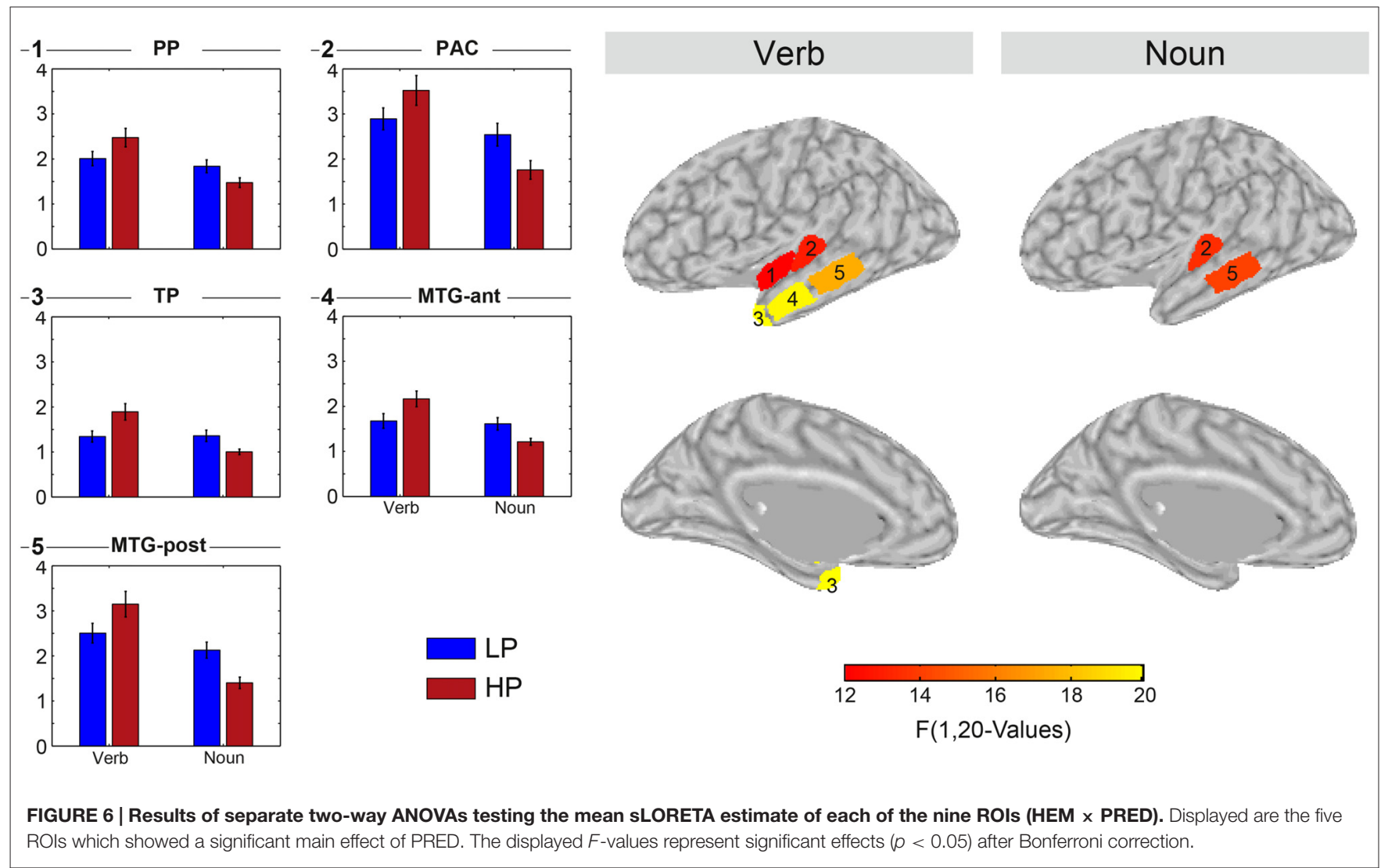

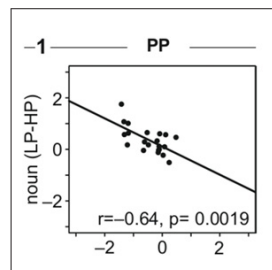
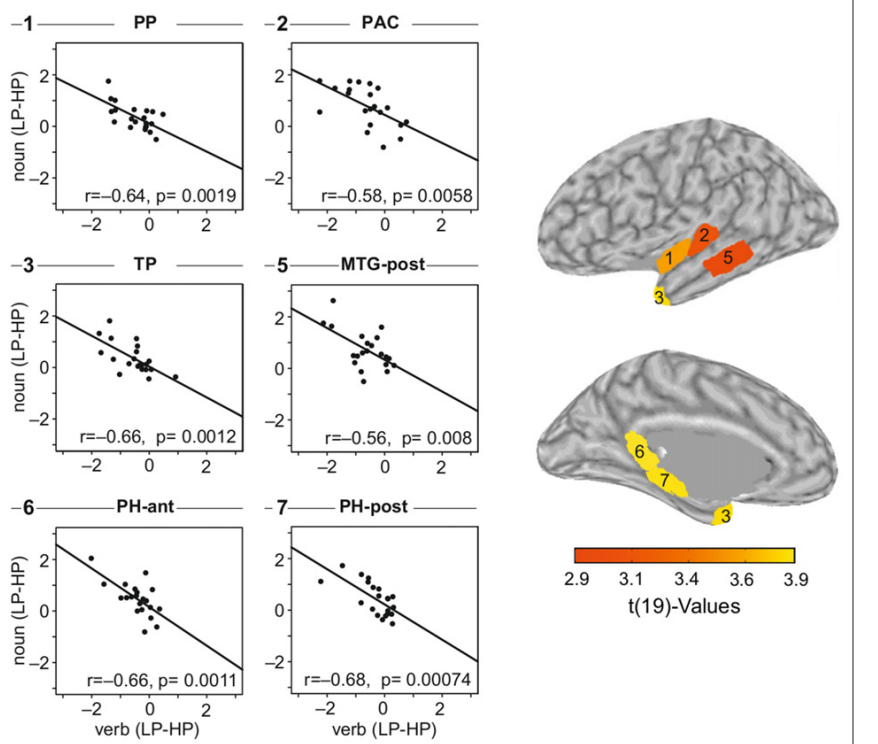

FIGURE 7 | Correlation plots for the six ROls which showed a significant relation between the verb- and the noun-effect. Effect sizes are estimated as the difference between the mean sLORETA estimates of the two conditions: LP-HP. The underlying data (before computing the difference) was just positive (magnitudes). The difference switches signs as HP elicited larger amplitudes during verb processing and LP during noun processing. and electroencephalography (EEG) or fMRI measurements (Gunter et al., 2000; Hald et al., 2007; Obleser and Kotz, 2010; Wang et al., 2012). Interestingly, when focusing on the prior verb we observed a reverse pattern: stronger N400 responses for the highly predictive (i.e., informative) vs. the less predictive verbs. Crucially, we found a negative correlation between the verb N400 effect and the noun N400 effect in left temporal cortex (PAC, PP, TP and pMTG) and also left parahippocampal area, suggesting an underlying functional relation.

The sLORETA estimates for the verbs and the nouns were very similar (Figure 5) pointing to a similarity in the functional domain. The classical N400 observed for unpredicted nouns and the N400 effect for the predictive verbs are both expressions of lexical-semantic processes indicated by a high correlation between the two. The observed N400 effect for the verbs did not depend on the integration of prior context, as we had provided just a minimal context of randomly using either of two personal pronouns (Sie vs. Er [She vs. He]). The verb N400 may thus represent differences in the semantic retrieval accounting for the differences in information for the two verb groups as a function of predictive power. Magnetic field and localized source topographies show that verbs and nouns activated a very similar network of semantic processes. So, one functional interpretation of the verb N400 may reflect a pre-activation of conceptual features due to semantic relatedness (Federmeier and Kutas, 1999; Van Petten et al., 1999; Kutas and Federmeier, 2000). In models of semantic memory, it is considered that there is a 
link between those items that share perceptual and functional features in common (Kutas and Federmeier, 2000; Patterson et al., 2007; Coutanche and Thompson-Schill, 2015). There is ample empirical evidence that mental representation of the verbs also include detailed grammatical and semantic information of the nominal components related to the verb (e.g., object; Shapiro et al., 1987, 1993; Friederici and Frisch, 2000; Li et al., 2006; Thompson et al., 2007). Therefore, semantic information of the verb impose selectional restrictions about the arguments, such as whether the arguments are animate or inanimate, human or nonhuman, instruments or non-instruments (Li et al., 2006). According to high-dimensional semantic space models derived from large-scale corpus analyses, such as Hyperspace Analogue to Language (HAL; (Burgess and Lund, 1997) and Latent Semantic Analysis (LSA; Landauer and Dumais, 1997), a word is defined by reference to the other words that co-occur with it. These co-occurrences can be encoded as a vector of weightings. Highly predictive verbs likely have strong weightings with a few specific co-occurrence items; consequently, less predictive verbs should have only weak co-occurrence weightings to other words. We assume that predictions are only made if evidence is sufficiently high. Therefore, processing of the predictive verbs likely lead to a co-activation of expected nouns, however, less predictive verbs do not cause multiple predictions for all possible continuations. Consequently, we hypothesize that during sentence processing the benefit of a confirmed prediction is paid earlier in time by stimulating the retrieval of semantic information. This is supported by the inverse correlation between the verb and the noun N400 effects. Forming predictions during sentence comprehension should be seen as a side effect of establishing semantic meaning while listening to the auditory input. If the recognized words allow a more rigid setup of the context, then this context is established immediately thereby creating a more restrictive prediction for the words to follow and thereby yielding an overall performance increase.

In terms of the source localization, the verb induced N400 effect demonstrated increased activation for the highly predictive vs. the less predictive verbs in mostly left hemispheric regions. Left hemispheric dominance was reported as well in another MEG study focusing on reading nouns in predictive contexts for latencies beyond $150 \mathrm{~ms}$, especially for the non-matching nouns (Dikker and Pylkkänen, 2013). More specifically, our results demonstrated a network of temporal and parahippocampal regions to be involved in prediction related processing of the $\mathrm{N} 400$ effect. In contrast to the neural sources found for a classical N400 effect, we did not observe left IFG to be part of the predictive processing network. Left IFG has been found important for selection of lexical candidates (ThompsonSchill et al., 1997, 1999; Lau et al., 2008). Therefore, one could have expected to observe a negative correlation between verb and noun N400 effects in the left IFG, if the predictive process was considered to be a controlled selection process. However, our data did not show involvement of the left IFG for predictive processing suggesting that the observed process was rather based on automatic co-occurrences than on a controlled process. For the left anterior temporal cortex, our results are consistent with Lau et al. (2016) who found this area to be most relevant for top-down facilitation of lexical-semantic predictions. Our results are also in line with those of Bonhage et al. (2015) who observed distributed temporal sources and no IFG activity for word specific predictions using fMRI. Finally, a prominent predictive effect was found in parahippocampal regions, which has been reported for linguistic processing (Meyer et al., 2005; Tracy and Boswell, 2008; Duff and Brown-Schmidt, 2012) and for predictive processing in general (Schiffer et al., 2012). Taken together, we interpret the fact that areas known to be involved in the classical N400 generation were also involved in predicting upcoming nouns as indicative for predictive coding (Bonhage et al., 2015).

The present results may allow us to formulate a simplified version of the predictive coding model on the N400 generation: let's presume that the processing of word B (e.g., car) depends on whether the preceding context word $\mathrm{A}$ is highly predictive $\left(A_{\mathrm{p}}\right.$; e.g., drive) or not $\left(A_{n}\right.$; e.g., get $)$. The predictive coding model assumes that $A_{\mathrm{p}}$ (e.g., drive) generates a prediction $\widetilde{\mathrm{B}}$ for the word B (e.g., car) with connections conveying $\widetilde{\mathrm{B}}$ from higher to lower cortical hierarchies. Processing of word B (e.g., car) would then require only the forward processing of the difference $B-\widetilde{B}$ up the processing hierarchy. The smaller the difference between prediction $\widetilde{B}$ and actual input $B$ (e.g., car), the lesser bottom-up information transfer and lesser adjustment at the higher level is required. In contrast, when word $A_{n}$ (e.g., get) does not allow generating predictions $\widetilde{\mathrm{B}}$, all information in word $\mathrm{B}$ (e.g., car) has to be conveyed from lower to higher levels. In summary, the processing of word B (e.g., car) and the internal information transfers much depend on the processing of the preceding context word A (e.g., drive or get), its specific information content and its relation to the target word B (e.g., car).

\section{CONCLUSION}

In summary, in the present sentence comprehension experiment we demonstrate that processing of predictive verbs as compared to less predictive verbs, evokes a response which is very similar to the classical N400 response in its temporal and distributional parameters. The observed N400 was larger for the predictive (i.e., informative) verbs than for the less predictive verbs. The predictive-verb N400 and the less predicted-noun N400 was inversely correlated which demonstrates a direct trade-off in terms of neural expenditure between the predictive and the predicted stage in lexical-semantic domain during language comprehension. The finding that evoked responses by the verbs were overall stronger than those by the nouns appears to reflect that, in general, verbs carry more information than nouns, thereby supporting the view that the N400 amplitude matches the amount of processed information. Our suggestion for a generalized functional interpretation of the N400 effect is thus that it reflects the effort to establish semantic meaning from perceived information.

\section{AUTHOR CONTRIBUTIONS}

BM and FM: experiment design, data collection, data analysis, article preparation; JO and ADF: experiment design, data 
analysis, article preparation; LH: data analysis, article preparation.

\section{ACKNOWLEDGMENTS}

This research was supported by the Max Planck Society (ADF, JO, BM, FM), Munich, Germany, the International Max Planck

\section{REFERENCES}

Bar, M. (2007). The proactive brain: using analogies and associations to generate predictions. Trends Cogn. Sci. 11, 280-289. doi: 10.1016/j.tics.2007.05.005

Bar, M. (2009). Predictions: a universal principle in the operation of the human brain. Introduction. Philos. Trans. R. Soc. Lond. B Biol. Sci. 364, 1181-1182. doi: $10.1098 /$ rstb.2008.0321

Bendixen, A., Schröger, E., and Winkler, I. (2009). I heard that coming: eventrelated potential evidence for stimulus-driven prediction in the auditory system. J. Neurosci. 29, 8447-8451. doi: 10.1523/JNEUROSCI.1493-09.2009

Bonhage, C. E., Müller, J. L., Friederici, A. D., and Fiebach, C. J. (2015). Combined eye tracking and fMRI reveals neural basis of linguistic predictions during sentence comprehension. Cortex 68, 33-47. doi: 10.1016/j.cortex.2015.04.011

Bonte, M., Parviainen, T., Hytönen, K., and Salmelin, R. (2006). Time course of top-down and bottom-up influences on syllable processing in the auditory cortex. Cereb. Cortex 16, 115-123. doi: 10.1093/cercor/bhi091

Brusini, P., Brun, M., Brunet, L., and Christophe, A. (2015). Listeners exploit syntactic structure on-line to restrict their lexical search to a subclass of verbs. Front. Psychol. 6:1841. doi: 10.3389/fpsyg.2015.01841

Burgess, C., and Lund, K. (1997). "Representing abstract words and emotional connotation in a high-dimensional memory space," in Proceedings of the Cognitive Science Society, (Hillsdale, NJ: Erlbaum), 61-66.

Coutanche, M. N., and Thompson-Schill, S. L. (2015). Creating concepts from converging features in human cortex. Cereb. Cortex 25, 2584-2593. doi: 10. 1093/cercor/bhu057

Dale, A. M., Fischl, B., and Sereno, M. I. (1999). Cortical surface-based analysis. I. Segmentation and surface reconstruction. Neuroimage 9, 179-194. doi: 10. 1006/nimg.1998.0395

Dambacher, M., Kliegl, R., Hofmann, M., and Jacobs, A. M. (2006). Frequency and predictability effects on event-related potentials during reading. Brain Res. 1084, 89-103. doi: 10.1016/j.brainres.2006.02.010

David, O., Maess, B., Eckstein, K., and Friederici, A. D. (2011). Dynamic causal modeling of subcortical connectivity of language. J. Neurosci. 31, 2712-2717. doi: 10.1523/JNEUROSCI.3433-10.2011

DeLong, K. A., Urbach, T. P., and Kutas, M. (2005). Probabilistic word pre-activation during language comprehension inferred from electrical brain activity. Nat. Neurosci. 8, 1117-1121. doi: 10.1038/nn1504

Demberg, V., and Keller, F. (2008). Data from eye-tracking corpora as evidence for theories of syntactic processing complexity. Cognition 109, 193-210. doi: 10 . 1016/j.cognition.2008.07.008

Destrieux, C., Fischl, B., Dale, A., and Halgren, E. (2010). Automatic parcellation of human cortical gyri and sulci using standard anatomical nomenclature. Neuroimage 53, 1-15. doi: 10.1016/j.neuroimage.2010.06.010

Dikker, S., and Pylkkanen, L. (2011). Before the N400: effects of lexical-semantic violations in visual cortex. Brain Lang. 118, 23-28. doi: 10.1016/j.bandl.2011. 02.006

Dikker, S., and Pylkkänen, L. (2013). Predicting language: MEG evidence for lexical preactivation. Brain Lang. 127, 55-64. doi: 10.1016/j.bandl.2012.08.004

Duff, M. C., and Brown-Schmidt, S. (2012). The hippocampus and the flexible use and processing of language. Front. Hum. Neurosci. 6:69. doi: 10.3389/fnhum. 2012.00069

Engel, A. K., Fries, P., and Singer, W. (2001). Dynamic predictions: oscillations and synchrony in top-down processing. Nat. Rev. Neurosci. 2, 704-716. doi: 10. $1038 / 35094565$

Federmeier, K. D. (2007). Thinking ahead: the role and roots of prediction in language comprehension. Psychophysiology 44, 491-505. doi: 10.1111/j.14698986.2007.00531.x
Research School on neuroscience of communication (FM), Leipzig, Germany, and Elekta Oy, Helsinki, Finland. Yvonne Wolff acquired the data. We would like to thank Jane Neumann and Helga Smallwood for valuable comments on manuscript preparation, Samu Taulu and Antti Ahonen for providing us with the advanced sensor noise suppression method for MEG data analysis.

Federmeier, K. D., and Kutas, M. (1999). A rose by any other name: long-term memory structure and sentence processing. J. Mem. Lang. 41, 469-495. doi: 10 1006/jmla.1999.2660

Federmeier, K. D., Wlotko, E. W., De Ochoa-Dewald, E., and Kutas, M. (2007). Multiple effects of sentential constraint on word processing. Brain Res. 1146, 75-84. doi: 10.1016/j.brainres.2006.06.101

Friederici, A. D. (2002). Towards a neural basis of auditory sentence processing. Trends Cogn. Sci. 6, 78-84. doi: 10.1016/s1364-6613(00) 01839-8

Friederici, A. D. (2011). The brain basis of language processing: from structure to function. Physiol. Rev. 91, 1357-1392. doi: 10.1152/physrev.00006.2011

Friederici, A. D., and Frisch, S. (2000). Verb argument structure processing: the role of verb-specific and argument-specific information. J. Mem. Lang. 43, 476-507. doi: 10.1006/jmla.2000.2709

Friston, K. (2003). Learning and inference in the brain. Neural Netw. 16, 1325-1352. doi: 10.1016/j.neunet.2003.06.005

Fruchter, J., Linzen, T., Westerlund, M., and Marantz, A. (2015). Lexical preactivation in basic linguistic phrases. J. Cogn. Neurosci. 27, 1912-1935. doi: 10.1162/jocn_a_00822

Gagnepain, P., Henson, R. N., and Davis, M. H. (2012). Temporal predictive codes for spoken words in auditory cortex. Curr. Biol. 22, 615-621. doi: 10.1016/j.cub. 2012.02.015

Gramfort, A., Luessi, M., Larson, E., Engemann, D. A., Strohmeier, D. Brodbeck, C., et al. (2013). MEG and EEG data analysis with MNE-Python. Front. Neurosci. 7:267. doi: 10.3389/fnins.2013.00267

Gramfort, A., Luessi, M., Larson, E., Engemann, D. A., Strohmeier, D., Brodbeck, C., et al. (2014). MNE software for processing MEG and EEG data. Neuroimage 86, 446-460. doi: 10.1016/j.neuroimage.2013.10.027

Griffiths, T. L., and Tenenbaum, J. B. (2011). Predicting the future as Bayesian inference: people combine prior knowledge with observations when estimating duration and extent. J. Exp. Psychol. Gen. 140, 725-743. doi: 10.1037/a 0024899

Gunter, T. C., Friederici, A. D., and Schriefers, H. (2000). Syntactic gender and semantic expectancy: ERPs reveal early autonomy and late interaction. J. Cogn. Neurosci. 12, 556-568. doi: 10.1162/089892900562336

Hald, L. A., Steenbeek-Planting, E. G., and Hagoort, P. (2007). The interaction of discourse context and world knowledge in online sentence comprehension. Evidence from the N400. Brain Res. 1146, 210-218. doi: 10.1016/j.brainres. 2007.02.054

Hale, J. (2001). "A probabilistic Earley parser as a psycholinguistic model," in Proceedings of the Second Meeting of the North American Chapter of the Association for Computational Linguistics on Language Technologies, (Stroudsburg, PA: Association for Computational Linguistics). 1-8

Halgren, E., Dhond, R. P., Christensen, N., Van Petten, C., Marinkovic, K., Lewine, J. D., et al. (2002). N400-like magnetoencephalography responses modulated by semantic context, word frequency and lexical class in sentences. Neuroimage 17, 1101-1116. doi: 10.1006/nimg.2002.1268

Halgren, E., Wang, C., Schomer, D. L., Knake, S., Marinkovic, K., Wu, J., et al. (2006). Processing stages underlying word recognition in the anteroventral temporal lobe. Neuroimage 30, 1401-1413. doi: 10.1016/j.neuroimage.2005. 10.053

Hämäläinen, M. S., and Ilmoniemi, R. J. (1994). Interpreting magnetic-fields of the brain-minimum norm estimates. Med. Biol. Eng. Comput. 32, 35-42. doi: 10. 1007/bf02512476

Hickok, G. (2012). The cortical organization of speech processing: feedback control and predictive coding the context of a dual-stream model. J. Commun. Disord. 45, 393-402. doi: 10.1016/j.jcomdis.2012.06.004 
Huang, Y. P., and Rao, R. P. N. (2011). Predictive coding. Wiley Interdiscip. Rev. Cogn. Sci. 2, 580-593. doi: 10.1002/wcs. 142

Jakuszeit, M., Kotz, S. A., and Hasting, A. S. (2013). Generating predictions: lesion evidence on the role of left inferior frontal cortex in rapid syntactic analysis. Cortex 49, 2861-2874. doi: 10.1016/j.cortex.2013.05.014

Kiebel, S. J., Daunizeau, J., and Friston, K. J. (2009). Perception and hierarchical dynamics. Front. Neuroinform. 3:20. doi: 10.3389/neuro.11.020.2009

Kutas, M., and Federmeier, K. D. (2000). Electrophysiology reveals semantic memory use in language comprehension. Trends Cogn. Sci. 4, 463-470. doi: 10. 1016/s1364-6613(00)01560-6

Kutas, M., and Federmeier, K. D. (2011). Thirty years and counting: finding meaning in the N400 component of the event-related brain potential (ERP). Annu. Rev. Psychol. 62, 621-647. doi: 10.1146/annurev.psych.093008. 131123

Kutas, M., and Hillyard, S. A. (1980). Reading senseless sentences-brain potentials reflect semantic incongruity. Science 207, 203-205. doi: 10.1126/science. 7350657

Kutas, M., and Hillyard, S. A. (1984). Brain potentials during reading reflect word expectancy and semantic association. Nature 307, 161-163. doi: 10. 1038/307161a0

Kutas, M., and Hillyard, S. A. (1989). An electrophysiological probe of incidental semantic association. J. Cogn. Neurosci. 1, 38-49. doi: 10.1162/jocn.1989. 1.1 .38

Landauer, T. K., and Dumais, S. T. (1997). A solution to Plato's problem: the latent semantic analysis theory of acquisition, induction and representation of knowledge. Psychol. Rev. 104, 211-240. doi: 10.1037/0033-295x.104. 2.211

Lau, E. F., Almeida, D., Hines, P. C., and Poeppel, D. (2009). A lexical basis for N400 context effects: evidence from MEG. Brain Lang. 111, 161-172. doi: 10. 1016/j.bandl.2009.08.007

Lau, E. F., Gramfort, A., Hämäläinen, M. S., and Kuperberg, G. R. (2013a). Automatic semantic facilitation in anterior temporal cortex revealed through multimodal neuroimaging. J. Neurosci. 33, 17174-17181. doi: 10. 1523/JNEUROSCI.1018-13.2013

Lau, E. F., Holcomb, P. J., and Kuperberg, G. R. (2013b). Dissociating N400 effects of prediction from association in single-word contexts. J. Cogn. Neurosci. 25, 484-502. doi: 10.1162/jocn_a_00328

Lau, E. F., Phillips, C., and Poeppel, D. (2008). A cortical network for semantics: (de)constructing the N400. Nat. Rev. Neurosci. 9, 920-933. doi: 10. $1038 / \mathrm{nrn} 2532$

Lau, E. F., Weber, K., Gramfort, A., Hämäläinen, M. S., and Kuperberg, G. R. (2016). Spatiotemporal signatures of lexical-semantic prediction. Cereb. Cortex 26, 1377-1387. doi: 10.1093/cercor/bhu219

Levy, R. (2008). Expectation-based syntactic comprehension. Cognition 106, 1126-1177. doi: 10.1016/j.cognition.2007.05.006

Lewis, A. G., and Bastiaansen, M. (2015). A predictive coding framework for rapid neural dynamics during sentence-level language comprehension. Cortex 68, 155-168. doi: 10.1016/j.cortex.2015.02.014

Lewis, A. G., Schoffelen, J. M., Schriefers, H., and Bastiaansen, M. (2016). A predictive coding perspective on beta oscillations during sentence-level language comprehension. Front. Hum. Neurosci. 10:85. doi: 10.3389/fnhum. 2016.00085

Li, X., Shu, H., Liu, Y., and Li, P. (2006). Mental representation of verb meaning: behavioral and electrophysiological evidence. J. Cogn. Neurosci. 18, 1774-1787. doi: $10.1162 /$ jocn.2006.18.10.1774

Maess, B., Herrmann, C. S., Hahne, A., Nakamura, A., and Friederici, A. D. (2006). Localizing the distributed language network responsible for the N400 measured by MEG during auditory sentence processing. Brain Res. 1096, 163-172. doi: 10. 1016/j.brainres.2006.04.037

Marinkovic, K., Dhond, R. P., Dale, A. M., Glessner, M., Carr, V., and Halgren, E. (2003). Spatiotemporal dynamics of modality-specific and supramodal word processing. Neuron 38, 487-497. doi: 10.1016/s0896-6273(03)00197-1

Maris, E., and Oostenveld, R. (2007). Nonparametric statistical testing of EEG- and MEG-data. J. Neurosci. Methods 164, 177-190. doi: 10.1016/j.jneumeth.2007. 03.024

Meyer, P., Mecklinger, A., Grunwald, T., Fell, J., Elger, C. E., and Friederici, A. D. (2005). Language processing within the human medial temporal lobe. Hippocampus 15, 451-459. doi: 10.1002/hipo.20070
Obleser, J., and Kotz, S. A. (2010). Expectancy constraints in degraded speech modulate the language comprehension network. Cereb. Cortex 20, 633-640. doi: 10.1093/cercor/bhp128

Obleser, J., and Kotz, S. A. (2011). Multiple brain signatures of integration in the comprehension of degraded speech. Neuroimage 55, 713-723. doi: 10.1016/j. neuroimage.2010.12.020

Oldfield, R. C. (1971). The assessment and analysis of handedness: the Edinburgh inventory. Neuropsychologia 9, 97-113. doi: 10.1016/0028-3932(71) 90067-4

Park, H., Ince, R. A., Schyns, P. G., Thut, G., and Gross, J. (2015). Frontal top-down signals increase coupling of auditory low-frequency oscillations to continuous speech in human listeners. Curr. Biol. 25, 1649-1653. doi: 10.1016/j.cub.2015. 04.049

Pascual-Marqui, R. D. (2002). Standardized low-resolution brain electromagnetic tomography (sLORETA): technical details. Methods Find. Exp. Clin. Pharmacol. 24, 5-12.

Patterson, K., Nestor, P. J., and Rogers, T. T. (2007). Where do you know what you know? The representation of semantic knowledge in the human brain. Nat. Rev. Neurosci. 8, 976-987. doi: 10.1038/nrn2277

Poeppel, D., Idsardi, W. J., and van Wassenhove, V. (2008). Speech perception at the interface of neurobiology and linguistics. Philos. Trans. R. Soc. Lond. B Biol. Sci. 363, 1071-1086. doi: 10.1098/rstb.2007.2160

Price, C. J. (2010). The anatomy of language: a review of 100 fMRI studies published in 2009. Ann. N Y Acad. Sci. 1191, 62-88. doi: 10.1111/j.1749-6632. 2010.05444.x

Pulvermüller, F., Shtyrov, Y., and Ilmoniemi, R. (2005). Brain signatures of meaning access in action word recognition. J. Cogn. Neurosci. 17, 884-892. doi: $10.1162 / 0898929054021111$

Pylkkänen, L., and Marantz, A. (2003). Tracking the time course of word recognition with MEG. Trends Cogn. Sci. 7, 187-189. doi: 10.1016/s13646613(03)00092-5

Pylkkänen, L., and McElree, B. (2007). An MEG study of silent meaning. J. Cogn. Neurosci. 19, 1905-1921. doi: 10.1162/jocn.2007.19.11.1905

Pylkkänen, L., Stringfellow, A., and Marantz, A. (2002). Neuromagnetic evidence for the timing of lexical activation: an MEG component sensitive to phonotactic probability but not to neighborhood density. Brain Lang. 81, 666-678. doi: 10. 1006/brln.2001.2555

Rao, R. P. N., and Ballard, D. H. (1999). Predictive coding in the visual cortex: a functional interpretation of some extra-classical receptive-field effects. Nat. Neurosci. 2, 79-87. doi: $10.1038 / 4580$

Rauss, K., Schwartz, S., and Pourtois, G. (2011). Top-down effects on early visual processing in humans: a predictive coding framework. Neurosci. Biobehav. Rev. 35, 1237-1253. doi: 10.1016/j.neubiorev.2010.12.011

Salmelin, R. (2007). Clinical neurophysiology of language: the MEG approach. Clin. Neurophysiol. 118, 237-254. doi: 10.1016/j.clinph.2006. 07.316

Schiffer, A.-M., Ahlheim, C., Wurm, M. F., and Schubotz, R. I. (2012). Surprised at all the entropy: hippocampal, caudate and midbrain contributions to learning from prediction errors. PLoS One 7:e36445. doi: 10.1371/journal.pone.00 36445

Shapiro, L. P., Gordon, B., Hack, N., and Killackey, J. (1993). Verb-argument structure processing in complex sentences in Broca/s and Wernicke's aphasia. Brain Lang. 45, 423-447. doi: 10.1006/brln.1993.1053

Shapiro, L. P., Zurif, E., and Grimshaw, J. (1987). Sentence processing and the mental representation of verbs. Cognition 27, 219-246. doi: 10.1016/s00100277(87)80010-0

Smith, N. J., and Levy, R. (2013). The effect of word predictability on reading time is logarithmic. Cognition 128, 302-319. doi: 10.1016/j.cognition.2013. 02.013

Sohoglu, E., Peelle, J. E., Carlyon, R. P., and Davis, M. H. (2012). Predictive top-down integration of prior knowledge during speech perception. J. Neurosci. 32, 8443-8453. doi: 10.1523/JNEUROSCI.5069-11.2012

Strauß, A., Kotz, S. A., and Obleser, J. (2013). Narrowed expectancies under degraded speech: revisiting the N400. J. Cogn. Neurosci. 25, 1383-1395. doi: 10. 1162/jocn_a_00389

Taulu, S., Simola, J., Kajola, M., Helle, L., Ahonen, A., Sarvas, J. (2012). "Suppression of uncorrelated sensor noise and artifacts in multi-channel MEG data," in 18th International Conference on Biomagnetism, Paris, 285. 
Taulu, S., Simola, J., and Kajola, M. (2005). Applications of the signal space separation method. IEEE Trans. Sign. Proc. 53, 3359-3372. doi: 10.1109/tsp. 2005.853302

Taylor, W. L. (1953). "Cloze Procedure”: a new tool for measuring readability. Journal. Q. 30, 415-433.

Thompson, C. K., Bonakdarpour, B., Fix, S. C., Blumenfeld, H. K., Parrish, T. B., Gitelman, D. R., et al. (2007). Neural correlates of verb argument structure processing. J. Cogn. Neurosci. 19, 1753-1767. doi: 10.1162/jocn.2007.19. 11.1753

Thompson-Schill, S. L., D’Esposito, M., Aguirre, G. K., and Farah, M. J. (1997). Role of left inferior prefrontal cortex in retrieval of semantic knowledge: a reevaluation. Proc. Natl. Acad. Sci. U S A 94, 14792-14797. doi: 10.1073/pnas. 94.26.14792

Thompson-Schill, S. L., D’Esposito, M., and Kan, I. P. (1999). Effects of repetition and competition on activity in left prefrontal cortex during word generation. Neuron 23, 513-522. doi: 10.1016/s0896-6273(00)80804-1

Tracy, J. I., and Boswell, S. B. (2008). "Mesial temporal lobe epilepsy: a model for understanding the relationship between language and memory," in Handbook of the Neuroscience of Language, eds B. Stemmer and H. A. Whitaker (Elsevier: UK), 319-328. doi: 10.1016/b978-008045352-1.00031-8

Van Petten, C., Coulson, S., Rubin, S., Plante, E., and Parks, M. (1999). Time course of word identification and semantic integration in spoken language. J. Exp. Psychol. Learn. Mem. Cogn. 25, 394-417. doi: 10.1037/0278-7393. 25.2.394

Van Petten, C., and Luka, B. J. (2012). Prediction during language comprehension: benefits, costs and ERP components. Int. J. Psychophysiol. 83, 176-190. doi: 10. 1016/j.ijpsycho.2011.09.015
Wahl, M., Marzinzik, F., Friederici, A. D., Hahne, A., Kupsch, A., Schneider, G. H., et al. (2008). The human thalamus processes syntactic and semantic language violations. Neuron 59, 695-707. doi: 10.1016/j.neuron.2008.07.011

Wang, L., Zhu, Z., and Bastiaansen, M. (2012). Integration or predictability? A further specification of the functional role of gamma oscillations in language comprehension. Front. Psychol. 3:187. doi: 10.3389/fpsyg.2012. 00187

Wlotko, E. W., and Federmeier, K. D. (2012). So that's what you meant! Eventrelated potentials reveal multiple aspects of context use during construction of message-level meaning. Neuroimage 62, 356-366. doi: 10.1016/j.neuroimage. 2012.04 .054

Conflict of Interest Statement: The authors declare that the research was conducted in the absence of any commercial or financial relationships that could be construed as a potential conflict of interest.

The reviewer ES and handling Editor declared their shared affiliation, and the handling Editor states that the process nevertheless met the standards of a fair and objective review.

Copyright (c) 2016 Maess, Mamashli, Obleser, Helle and Friederici. This is an open-access article distributed under the terms of the Creative Commons Attribution License (CC BY). The use, distribution and reproduction in other forums is permitted, provided the original author(s) or licensor are credited and that the original publication in this journal is cited, in accordance with accepted academic practice. No use, distribution or reproduction is permitted which does not comply with these terms. 